\title{
Clinical utility of cytomegalovirus urine cultures for ophthalmic care in patients with HIV
}

\author{
Marcus-M Gellrich, Elisabeth Baumert, Jörg A Rump, Peter Vaith, Frank T Hufert, \\ Lutz L Hansen
}

\begin{abstract}
Background-The utility of cytomegalovirus (CMV) urine cultures was checked in patients with HIV (a) to identify those at risk for CMV retinitis and (b) to guide clinical decisions on treatment and prophylaxis of $\mathrm{CMV}$ retinitis.

Methods-HIV infected patients were tested for CMVuria by shell vial cell cultures. The prevalence of CMVuria was related to CD4 count, HIV risk group, and time before and after diagnosis of $\mathrm{CMV}$ retinitis
\end{abstract}

Results-A total of 639 shell vial cell cultures were obtained from 266 HIV infected ophthalmic patients. Only $4 \%$ of all patients with a CD4 count $>400 \times 10^{6} / 1$ shed CMV in their urine compared with $42 \%$ with a CD4 count $\leq 50 \times 10^{6} / 1$. Twenty three of 25 patients with $C M V$ retinitis had a CD4 count $\leq 50 \times 10^{6} / 1$. Among 130 patients with a CD4 count $\leq 50 \times 10^{6} / 1$ (a) those who were CMVuric had a nearly sevenfold risk (p<0.0001) of developing CMV retinitis (35\%) compared with those who did not shed CMV in their urine (5\%), and (b) CMVuria and CMV retinitis were more frequent in homosexuals $(58 \% / 25 \%)$ than in intravenous drug users $(23 \% / 15 \%)$. More than 1 year before diagnosis of $\mathrm{CMV}$ retinitis $18 \%$ of patients were CMVuric compared with $83 \%$ of patients who were CMV culture positive in the last 3 months. CMVuria under virustatic maintenance Universitäts-Augenklinik, therapy is associated with worsening of Universität Freiburg, Germany M-M Gellrich

E Baumert

L L Hansen

Medizinische Klinik, Universität Freiburg, Germany

J A Rump

P Vaith

Institut für

Medizinische

Mikrobiologie der

Universität Freiburg,

Germany

F T Hufer

Correspondence to: Marcus-M Gellrich Universitäts-Augenklinik, Killianstrasse 5D-79106, Freiburg, Germany.

Accepted for publication 28 June 1996 retinitis in two thirds of cases. Conclusion-Ophthalmic screening of patients with HIV should include those with a CD4 count $\leq 50 \times 10^{6} / 1$ and focus on the subgroup with additional CMVuria. Screening of other patients can be dropped without undue risk in order to spare AIDS patients unnecessary hospital visits. CMVuria as a single finding, however, does not justify antiviral prophylaxis of CMV retinitis.

(Br F Ophthalmol 1996;80:818-822)

Cytomegalovirus (CMV) retinitis is by far the most important disease of the eye in AIDS as it leads to blindness if not treated in time. The main task for the ophthalmologist in HIV infection is to recognise this blinding disease early and at least guide the antiviral treatment. Screening of AIDS patients for CMV retinitis would, therefore, be indicated. ${ }^{1-5}$ AIDS patients, however, have to endure frequent visits to different medical specialists during the final phase of their life. Thus, it would be helpful to know what factors indicate a high risk for developing CMV retinitis. ${ }^{6}$ This would not only be useful in reducing visits to the ophthalmologist but could also help in deciding whether to use antiviral prophylaxis. At present three markers seem to be candidates for recognising the risk of getting CMV retinitis: the number of CD4 lymphocytes in the blood (CD4 count), the presence of CMVuria ${ }^{6}{ }^{6}$ and the CMV pp65 antigen test. ${ }^{7}$

The CD4 count is a well established laboratory marker for the risk of developing opportunistic disease in HIV infection. ${ }^{8}$ Thus, chemoprophylaxis for Pneumocystis carinii pneumonia ${ }^{9}$ is recommended at $\mathrm{CD} 4$ counts lower than $200 \times 10^{6} / 1$ and for cerebral toxoplasmosis at CD4 counts less than $100 \times 10^{6} / 1$ with a positive test result for anti-toxoplasma IgG. ${ }^{10}$ CMV retinitis, however, occurs at much lower CD4 concentrations, usually below $50 \times 10^{6} / 1^{11}$ : from our clinical experience with many intravenous drug users among our HIV patients we felt that a CD4 count below $50 \times 10^{6} / 1$ as the only criterion for starting primary $\mathrm{CMV}$ prophylaxis ${ }^{12}$ would result in considerable overtreatment.

In this retrospective study we checked the usefulness of CMVuria in defining risk for CMV retinitis among those patients with CD4 counts lower than $50 \times 10^{6} / 1$. Furthermore, we evaluated CMVuria as a factor for the effectiveness of secondary prophylaxis in patients with CMV retinitis.

\section{Patients and methods}

We analysed retrospectively the data from 294 HIV patients referred to our hospital by our medical clinic (outpatient department or infective ward) between January 1990 and April 1995 for complete ophthalmic examination. This included visual acuity testing and slitlamp examination if possible but always funduscopy with dilated pupils. While all inpatients were screened routinely for eye involvement of HIV infection the basis for referral of outpatients was as follows: (1) all patients for an initial examination regardless of the stage of HIV infection; (2) all patients with ocular symptoms; and (3) all patients with CD4 counts less than $100 \times 10^{6} / \mathrm{l}$ every 3 months.

The patients were staged according to the CDC classification ${ }^{13}$ and got regular CD4 counts (whole blood assay). Since 1991 they have also been checked routinely for cytomega- 


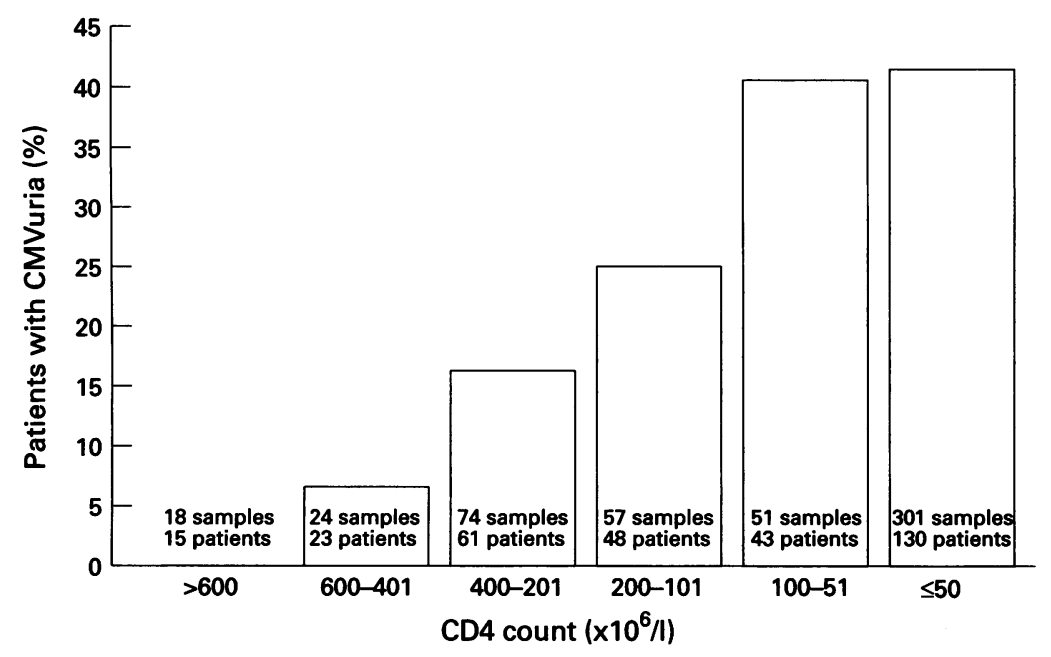

Figure 1 CD4 count and prevalence of CMVuria.

Figure 2 Prevalence of CMVuria before the diagnosis of $C M V$ retinitis.

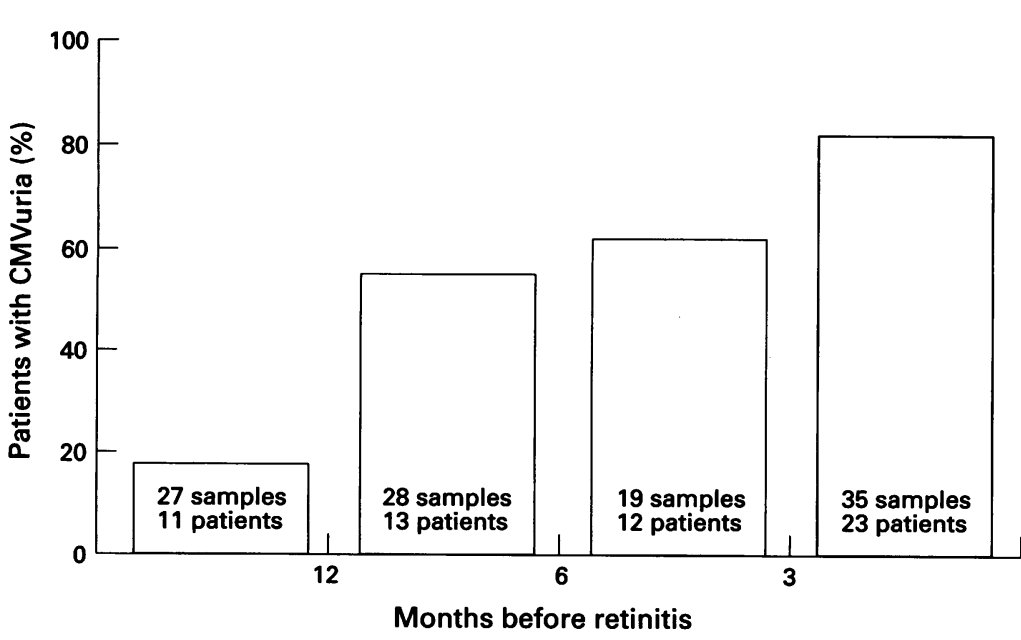

Table 1 Risk groups of HIV patients in Freiburg ( $n=266)$

\begin{tabular}{lrr}
\hline \multirow{2}{*}{ Risk group } & \multicolumn{2}{l}{ Patients } \\
\cline { 2 - 3 } & $n$ & $\%$ \\
\hline Homosexuals & 103 & 39 \\
Drug addicts & 86 & 32 \\
Heterosexuals & 31 & 12 \\
Africans & 11 & 4 \\
Haemophiliacs & 14 & 5 \\
Transfusion & 4 & 2 \\
Unknown & 17 & 6 \\
Total & 266 & 100 \\
\hline
\end{tabular}

«No information available on risk group.

risk groups see Table 1). While 639 shell vial cultures could be included in our study there were 208 ophthalmic examinations without a corresponding testing for CMVuria. The commonest reasons for cultures not being done were (1) ophthalmic examination before 1991 (when regular CMV testing was established), (2) fundus control within a short time (CMVuria was tested usually every 3 months), and (3) inability of outpatients to produce urine on that particular occasion.

The prevalence of CMVuria for different CD4 classes in patients not treated for CMV infection is shown in Figure 1 (266 patients, 525 urine cultures). All patients with a CD4 count $>600 \times 10^{6} / 1$ tested negative. Only $4 \%$ of all patients with a CD4 count $>400 \times 10^{6} / 1$ shed CMV in the urine compared with $21 \%$ of all patients with a CD4 count from 400 to $101 \times 10^{6} / 1$ There is, however, no great difference among patients with a $\mathrm{CD} 4$ count from 100 to $51 \times 10^{6} / 1$ and $\leq 50 \times 10^{6} / 1$ with $41 \%$ and $42 \%$, respectively, being positive for CMV in urine culture.

\section{CMVURIA AND CMV RETINITIS}

The prevalence of CMVuria and CMV retinitis in patients with a CD4 count $\leq 50 \times 10^{6} / 1$ is shown in Table 2 . The rate of retinitis was $18 \%$ in these patients with a higher but not significantly different percentage in homosexuals $(25 \%)$ than in intravenous drug users (15\%). CMVuria, on the other hand, was more frequent $(p<0.001)$ in homosexuals $(58 \%)$ than in intravenous drug users $(23 \%)$. Interestingly only two of 25 patients with CMV retinitis had a CD4 count $>50 \times 10^{6} / 1$ and 21 had CMVuria. Among all patients with a CD4 count $\leq 50 \times 10^{6} / 1$ those with CMVuria have a nearly sevenfold risk for CMV retinitis (19/54 $=35 \%$ ) compared with patients with a CD4 count $\leq 50 \times 10^{6} / 1$ without CMVuria $(4 / 76=5 \%$, $\mathrm{p}<0.0001)$. Among the subgroups of homo-

Table 2 Prevalence of CMV uria and CMV retinitis in different risk groups

\begin{tabular}{llll}
\hline & \multicolumn{3}{l}{ Patients with CD4 count $\leq 50 \times 10^{6} / l$} \\
\cline { 2 - 4 } & $\begin{array}{l}\text { All patients } \\
n(\%)\end{array}$ & $\begin{array}{l}\text { Homosexuals } \\
n(\%)\end{array}$ & $\begin{array}{l}\text { Drug misusers } \\
n(\%)\end{array}$ \\
\hline All & 130 & 59 & 39 \\
+ CMV retinitis & $23(18)$ & $15(25)$ & $6(15)^{\mathrm{ns}}$ \\
+ CMVuria & $54(42)$ & $34(58)$ & $9(23)^{\star}$ \\
+ CMVuria +CMV & $19(35)$ & $13(38)$ & $4(44)^{\dagger}$ \\
$\quad$ & & &
\end{tabular}

$n s=p \geq 0.05 ;{ }^{*} p<0.001$ homosexuals compared with drug misusers; tpercentage of patients with CMVuria. 
Table 3 CMV retinitis patients with CMVuria under intravenous virustatic therapy

\begin{tabular}{llll}
\hline & $A S$ & $K C$ & $S W$ \\
\hline Time after diagnosis & 14 weeks & 29 weeks & 11 weeks \\
Present weekly therapy & Foscarnet discontinued & Ganciclovir $3 \times 10 \mathrm{mg} / \mathrm{kg}$ & Ganciclovir $3 \times 10 \mathrm{mg} / \mathrm{kg}$ \\
Course of retinitis & No progression & New lesion: paramacular & Marked progression on both eyes \\
\hline
\end{tabular}

sexuals and intravenous drug users with a CD4 count $\leq 50 \times 10^{6} / 1$ the relative risk for $\mathrm{CMV}$ retinitis is still nearly five- and sevenfold, respectively $(\mathrm{p}<0.01)$.

All 25 patients with CMV retinitis had been checked once or several times preceding the occurrence of retinitis. In Figure 2 the percentage of patients who shed CMV in their urine is shown at the time before the diagnosis of CMV retinitis (109 urine samples, 25 patients). More than 1 year before the diagnosis positive cultures of CMV could be obtained in only $18 \%$ of the patients, while during the last 3 months before clinical manifestation of retinitis CMVuria was found in $83 \%$ of patients (19/23).

\section{CMVURIA UNDER VIRUSTATIC THERAPY}

Twenty three of 25 patients with CMV retinitis were started on intravenous therapy with either foscarnet or ganciclovir. One patient refused treatment and one patient who had peripheral retinal involvement in only one eye and was CMV negative in the urine only received intraocular injections of ganciclovir. In 21 patients 114 urine cultures for CMV were obtained after the initiation of intravenous antiviral therapy. At the beginning of treatment $19 / 21$ patients were CMV positive in the urine $(90 \%)$. During the first month of induction treatment $47 \%$ of urine cultures ( $14 / 30$ cultures in $8 / 16$ patients) were still positive for CMV, while thereafter this was found in only $5 \%$ (4/84 cultures in $3 / 18$ patients).

In two of these three patients the occurrence of CMVuria was associated with progression of retinitis under maintenance therapy with ganciclovir (Table 3). They received the reinduction dose whereafter retinitis stabilised again and CMV was eliminated from the urine.

\section{Discussion}

LIMITATIONS OF THE STUDY

Apart from the fact that our group of HIV patients is small in comparison with larger centres some other limitations of our study need to be discussed. Since our investigation is retrospective in nature there is no close monitoring of HIV patients for CMVuria. Thus, 208 examinations could not be related to a culture result while 639 urine cultures could be included in our study. There are, however, fewer than $10 \%$ of patients $(28 / 294)$ without even one urine examination for CMV in the study period.

Selection bias probably plays no major role for inpatients as they were nearly all examined ophthalmically. There was, however, a trend for outpatients to be sent more readily if they had positive urine cultures for CMV. Thus, the true prevalence for CMVuria in the different
CD4 classes might be slightly lower. This would, however, still increase the statistical risk for patients with CMVuria to develop CMV retinitis.

\section{CD4 COUNT AS INDICATOR FOR CMV RETINITIS SCREENING}

Often a devastating course of CMV retinitis can be prevented by screening of asymptomatic subjects and by early therapy. ${ }^{1}{ }^{2}{ }^{5}$ Several screening concepts have been offered so far although the efficacy of most of them has not been proved. While Fabricius ${ }^{3}$ suggests ophthalmic screening for all patients with AIDS, others advise regular examinations only for patients with CD4 counts $<200$ cells $\times 10^{6} / 1^{4}$ or $<50$ cells $\times 10^{6} / 1^{15}$

We feel that even this is a rather low threshold prompting many unnecessary visits of AIDS patients to the ophthalmologist. Indeed, $92 \%$ of our patients with CMV retinitis had CD4 counts $\leq 50 \times 10^{6} / 1$ which confirms the findings of others ${ }^{11}{ }^{16}$ that these patients are at particular risk. However, in our study only every fifth patient had a CMV retinitis (every fourth in a homosexual population), if one only takes into account the very low CD4 count of $\leq 50 \times 10^{6} / 1$. Also we saw no patient with $\mathrm{CMV}$ retinitis having a CD4 count $>200 \times 10^{6} / 1$ as has been reported. ${ }^{17}$ Based upon our data, we believe one should not rely only on the CD4 count as single indicator for screening.

CMVURIA AS AN INDICATOR FOR THE RISK OF CMV RETINITIS

In our study $83 \%$ of AIDS patients with CMV retinitis shed $\mathrm{CMV}$ in their urine during the last 3 months before diagnosis of retinitis. These data are similar to those of Zurlo et al ${ }^{6}$ who even found $19 / 19$ patients to have positive urine cultures during the 7 days before diagnosis of retinitis. In our series CMV retinitis occurred in $35 \%$ of all patients with both a CD4 count $\leq 50 \times 10^{6} / 1$ and CMVuria, indicating a nearly sevenfold risk of patients with CMVuria developing CMV retinitis in comparison with those without CMVuria.

If primary prophylaxis with oral ganciclovir is considered at all for HIV patients ${ }^{12}$ it seems worth while mainly to address the high risk group with a CD4 count $\leq 50 \times 10^{6} / 1$ and additional CMVuria. If we had screened only these patients, in 19 of 25 cases (76\%) CMV retinitis would have been diagnosed early. Of the six remaining cases two had higher CD4 counts and four were not CMV positive in the urine. Interestingly, these latter four patients had a more benign course of retinitis with no loss of central vision and no involvement of an eye which was primarily free of retinitis (3/8). Thus we agree with Zurlo et al ${ }^{6}$ who stated that viruria is sensitive in identifying a population 
that could have CMV disease, but we do not agree with their conclusion that CMVuria is not a helpful factor for clinical decisions.

Zurlo et $a l^{6}$ only looked at the possible use of CMVuria as a single predictor for clinical purposes. We could show, as did others, ${ }^{18} 19$ that the prevalence of CMVuria increases with falling CD4 count. There are many HIV patients, however, with a CD4 count $>50 \times 10^{6} / 1^{6}$ and even healthy people without HIV infection who shed CMV in their urine. ${ }^{20}$ Knowing that CMV retinitis occurs only in a few with higher CD4 counts ${ }^{17}$ we do not advise regular testing for $\mathrm{CMVuria}^{2}$ until the CD4 count has dropped below $50 \times 10^{6} / 1$. If a patient is tested positive we examine him every 6 weeks, if tested negative every 3 months.

With our screening concept we aim to reduce the frequency of visits of the AIDS patient in general without a substantial increase of the risk of an undetected CMV retinitis. Our concept results in different screening frequencies for different risk groups: homosexuals with a CD4 count $\leq 50 \times 10^{6} / 1$ were 2.5 times more likely to shed CMV in their urine than intravenous drug users ${ }^{20}$ and 1.7 times more likely to develop CMV retinitis. This difference among the risk groups cannot be attributed to greater immunosuppression in homosexuals $^{21}$ since our comparison refers only to HIV patients with a CD4 count $\leq 50 \times 10^{6} / 1$. It is obvious that, with our model, low risk groups for CMV retinitis will benefit most from a reduction in screening examinations. This is an important point in clinical work at our eye hospital with $32 \%$ intravenous drug users among our HIV patients, who are often difficult to motivate for follow up examinations.

Once intravenous therapy is started CMV is usually eliminated from the urine within a month ( $95 \%$ of urines becoming CMV negative). We presented three patients who became viruric again under intravenous therapy. In two of these patients there was considerable progression of retinitis making a new treatment regimen necessary. We therefore check retinitis patients under treatment at each visit for CMVuria and propose a change of therapy if they become positive again. Many of our patients, however, had progression of $\mathrm{CMV}$ retinitis but no CMV in the urine. Therefore, regular fundus controls in patients with retinitis may not be replaced by laboratory tests.

\section{OTHER CMV ANTIGEN TESTS}

Cytomegalovirus disease should be regarded as a systemic infection with different organ manifestations among which CMV retinitis is the commonest. ${ }^{22}$ From nine cultures of bronchoalveolar lavage (BAL) material which were done on our retinitis patients eight had results which agreed with urine cultures done at that time while only one patient was negative in urine culture and positive in BAL culture. Clearly from the ophthalmologist's point of view CMV cultures from urine have the advantage over other body fluids that they are easy to obtain and bear low HIV infectivity. ${ }^{23}$ Also Zurlo et al pointed out that during the 7 days before diagnosis of CMV retinitis 19/19 patients had a positive urine culture while only $14 / 23$ had a positive blood culture. ${ }^{6}$

For reasons not yet understood the sensitivity of cultures for the diagnosis of extraocular CMV disease seems to be much lower. Zurlo et $a l^{6}$ found only $2 / 5$ patients to be positive in urine and only $2 / 6$ patients to be positive in blood during the last week before diagnosis of CMV pneumonitis or CMV colitis. Here the CMV pp65 antigen test may have advantages for the early diagnosis of CMV disease because it was found to be more sensitive than shell vial cultures for detection of CMV in polymorphonuclear leucocytes. ${ }^{24}$ The CMV pp65 antigen test in contrast with conventional cultures gives quantitative information by counts of positive cells. ${ }^{7} \mathrm{~A}$ third sort of CMV antigen test, the polymerase chain reaction (PCR), ${ }^{7}$ was shown to be highly concordant with the CMV pp65 antigen test, ${ }^{25}$ but there is still the disadvantage that PCR is less rapid and simple than the CMV pp65 antigen test. ${ }^{7}$ One might expect, however, that the latter two antigen tests are less negatively influenced by antiviral treatment than the CMV culture technique.

Ophthalmologists caring for HIV patients know that early diagnosis of CMV retinitis is the key to preservation of vision and that screening of patients at risk is worth while. We have learned that regular eye examinations of all patients with AIDS are less effective than introducing a CD4 margin-for example, 50 cells $\times 10^{6} / 1$, below which screening should start. Clearly it is no longer sufficient to increase the screening frequency only in cases of extraocular manifestations of CMV disease. ${ }^{35}$ Ophthalmologists should instead demand regular systemic testing for CMV antigen in all HIV patients with a CD4 count below 50 cells $\times$ $10^{6} / 1 .^{24}$

At present a study is under way in our clinic comparing the usefulness of CMV-PCR, the CMV pp65 antigen test in blood, and CMVuria for ophthalmological screening. But as long as none of the tests available has proved yet to be a better predictor of retinitis we will continue to use the urine culture for the reasons mentioned above. Holding to this concept ophthalmologists may not need primary chemoprophylaxis for CMV retinitis.

1 Holland GN, Pepose JS, Pettit TH, Gottlieb MS, Yee RD, Foos RY. Acquired immune deficiency syndrome. Ocular manifestations. Ophthalmology 1983;90:859-73.

2 Salmon D, Lacassin F, Harzic M, Leport C, Perronne C, Bricaire $F$, et al. Predictive value of cytomegalovirus viraemia for the occurrence of CMV organ involvement in AIDS. $f$ Med Virol 1990;32:160-3.

3 Fabricius E-M. Augenmanifestationen bei HIV-Infektionen. Stuttgart: Enke, 1992

4 Garweg J. Opportunistische Augenerkrankungen im Rahmen der HIV-Infektion. Klin Monatsbl Augenheilkd 1993; 202:465-70.

5 Dhillon $B$. The management of cytomegalovirus retinitis in AIDS. Br $\mathcal{f}$ Ophthalmol 1994;78:66-9.

6 Zurlo J, O'Neill D, Polis MA, Manischewitz J, Yarchoan R, Zurlo J, O'Neill D, Polis MA, Manischewitz J, Yarchoan R,
Baseler M, et al. Lack of clinical utility of cytomegalovirus Baseler M, et al. Lack of clinical utility of cytomegalovirus
blood and urine cultures in patients with HIV infection. blood and urine cultures in patie
Ann Intern Med 1993;118:12-7.

7 Freymuth F, Gennetay E, Petitjean J, Eugene G, De Ligny $\mathrm{BH}$, Ryckelynck JP, et al. Comparison of nested PCR for detection of DNA in plasma with pp65 leukocytic antigenemia procedure for diagnosis of human cytomegalovirus infection. f Clin Microbiol 1994;32:1614-8.

8 Fauci AS. The human immunodeficiency virus. Infectivity and mechanisms of pathogenesis. Science 1988;239:61722. 
9 US Public Health Service Task Force on Antipneumocystis Prophylaxis in Patients with Human Immunodeficiency Virus Infection. Recommendations for prophylaxis against Pneumocystis carinii pneumonia for persons infected with human immunodeficiency virus. F Acquir Immun Defic Syndr 1993;6:46-55.

10 Gallant JE, Moore RD, Chaisson RE. Prophylaxis for opportunistic infections in patients with HIV infection. opportunistic infections in patien

11 Kuppermann BD, Petty JG, Richman DD, Mathews WC Fullerton SC, Rickman LS, et al. Correlation between $\mathrm{CD} 4+$ counts and prevalence of cytomegalovirus retinitis and human immunodeficiency virus-related noninfectious retinal vasculopathy in patients with acquired immunodeficiency syndrome. Am F Ophthalmol 1993;115:575-82.

12 McCarthy M. Oral ganciclovir fails to prevent CMV in HIV trial. Lancet 1995;346:895.

13 Centers for Disease Control. Classification system for human T-lymphotropic virus type III/lymphadenopathyassociated virus infections. Ann Intern Med 1986;105:234 7 .

14 Gleaves CA, Smith TF, Shuster EA, Pearson GR. Comparison of standard tube and shell vial cell culture techniques for the detection of cytomegalovirus in clinical specimens. f Clin Microbiol 1985;21:217-21.

15 The Oral Ganciclovir European and Australian Cooperative Study Group. Intravenous versus oral ganciclovir European/Australian comparative study of efficacy and safety in the prevention of cytomegalovirus retinits recurrence in patients with AIDS. AIDS 1995;9:471-7.

16 Palestine AG, Rodrigues MM, Macher AM, Chan CC Lane HC, Fauci AS. Ophthalmic involvement in acquired Lane HC, Fauci AS. Ophthalmic involvement in acquired 1092-9.

17 Crowe SM, Carlin JB, Stewart KI, Lucas CR, Hoy JF. Predictive value of $\mathrm{CD} 4+$ lymphocyte numbers for the devel- opment of opportunistic infections and malignancies in HIV-infected persons. F Acquir Immune Defic Syndr 1991;4 $770-6$

18 Drew WL, Mills J, Levy J, Dylewski J, Casavant C, Ammann $\mathrm{AJ}$, et al. Cytomegalovirus infection and abnormal T-lymphocyte subset ratios in homosexual men. Ann Intern Med 1985;103:61-6.

19 Gerna G, Parea M, Percivalle E, Zipeto D, Silini E, Barbarini $G$, et al. Human cytomegalovirus viraemia in HIV-1ini $\mathrm{G}$, et al. Human cytomegalovirus viraemia in $\mathrm{HIV}-1-$ seropositive patients at

20 Drew WL, Mintz L, Miner RC, Sands M, Ketterer B Prevalence of cytomegalovirus infection in homosexual men. $\mathcal{F}$ Infect Dis 1981;143:188-92.

21 Cheong I, Flegg PJ, Brettle RP, Welsby PD, Burns SM Dhillon B, et al. CMV disease in AIDS: the Edinburgh experience. Int $\mathcal{Y}$ STD and AIDS 1992;3:324-8.

22 Peters BS, Beck EJ, Anderson S, Coleman D, Coker R, Main J, et al. Cytomegalovirus infection in AIDS. Pattern of disease, response to therapy and trends in survival. $f$ Infect 1991;23:123-8.

23 Geier St A, Gürtler L, Klauss V, Mueller A, Bader L, Eberle $\mathrm{J}$, et al. Unterschied in der Nachweisbarkeit von Humanem Immunschwäche Virus Typ 1 in Tränenflüssigkeit und Blutlymphozyten. Klin Monatsbl Augenheilkd 1992;201: 164-8.

24 Erice A, Holm MA, Gill PC, Henry S, Dirksen CL, Dunn $\mathrm{DL}$, et al. Cytomegalovirus (CMV) antigenemia assay is more sensitive than shell vial cultures for rapid detection of CMV in polymorphonuclear blood leukocytes. $f$ Clin Microbiol 1992;30:2822-5.

25 Revello MG, Percivalle E, Sarasini A, Baldanti F, Furione M, Gerna G. Diagnosis of human cytomegalovirus infection of the nervous system by pp65 detection in polyinfection of the nervous system by pp65 detection in polyAIDS patients. F Infect Dis 1994;170:1275-9. 